\title{
Urine neopterin: a new parameter for serial monitoring of disease activity in patients with systemic lupus erythematosus
}

\author{
Kean L Lim, Ken Muir, Richard J Powell
}

\begin{abstract}
Objective-To investigate the role of serial measurement of urine neopterin concentration in monitoring the progression of systemic lupus erythematosus (SLE) disease activity scored using the British Isles Lupus Assessment Group (BILAG) index.

Methods-We followed prospectively 68 unselected SLE patients for a total of 464 patient months during which 233 separate assessments were carried out. At each assessment, urine neopterin, determined by high performance liquid chromatography, together with erythrocyte sedimentation rate (ESR) and plasma $\mathrm{C3}, \mathrm{C} 4$, and C3d were measured and the SLE disease activity scored by a single observer. Serial data sets were analysed using time series modelling techniques.

Results-Single time point analysis showed a significant increase in urine neopterin concentrations in 14 patients who suffered flares of their disease during the study period $(p=0 \cdot 02)$. Thirty patients with active disease went into disease remission with significant decreases in their urine neopterin values $(p=0.02)$. In the time series analysis, a statistically significant association was found between serial concentrations of urine neopterin and BILAG score $(r=0.6, p<0.05)$; no other study parameter (ESR and serum C3, C4, and C3d) mirrored SLE disease activity as effectively.

Conclusions-This study provides initial evidence that changes in urine concentrations of neopterin are significantly correlated with fluctuations in disease activity over time, scored using the BILAG index, amongst individual patients with SLE. Consequently, serial urine neopterin measurements appear to be clinically useful for monitoring disease activity and may contribute substantially to therapeutic decision making in these patients.
\end{abstract}

(Ann Rheum Dis 1994; 53: 743-748)

A challenge in the clinical management of patients with systemic lupus erythematosus (SLE) is to identify laboratory parameters that will distinguish those with active and those with inactive disease, and parameters that can be used to monitor serially disease activity and disease progression. Erythrocyte sedimentation rate (ESR), serum or plasma complement concentrations, and antibodies to double stranded DNA (dsDNA) are currently used in clinical practice as markers of disease activity. However, some patients can have persistently abnormal results in these tests and yet show few clinical symptoms or functional deterioration of a major organ, whilst other patients are severely ill with only minimal changes in these parameters. Moreover, some parameters are only useful in certain patterns of organ involvement in SLE; for example, changes in anti-dsDNA and complement concentrations are reported predominantly to accompany flares of lupus nephritis. ${ }^{12}$

Urine neopterin, an indirect marker of the upregulation of the cellular immune system, ${ }^{3}$ has previously been demonstrated to be of value as a parameter of disease activity in an unselected group of SLE patients. ${ }^{4}$ In that study patients with SLE, irrespective of disease activity, exhibited significantly increased concentrations of urine neopterin compared with healthy controls. Using logistic regression analysis, it was shown that urine neopterin was superior to serum soluble interleukin-2 (sIL-2) receptors and anti-dsDNA, ESR, and plasma complement $\mathrm{C} 3, \mathrm{C} 4$ and $\mathrm{C} 3$ degradation products $(\mathrm{C} 3 \mathrm{~d})$ as a predictor of disease activity in patients with SLE. Urine neopterin appeared to be a more reliable index of global clinical activity in SLE patients, irrespective of organ involvement or treatment with corticosteroid or cytotoxic agents, or both; however, the temporal relationship between urine neopterin and SLE disease activity was not addressed. This study investigated the role of serial urine neopterin in relationship to SLE disease activity scored using the British Isles Lupus Assessment Group (BILAG) index. ${ }^{5}$

\section{Patients and methods}

The study population consisted of 68 unselected patients regularly attending the connective tissue disease clinic who fulfilled four or more of the 1982 revised American Rheumatism Association criteria for the classification of SLE. ${ }^{6}$ The mean (SD) age and the mean (SD) disease duration of the patient cohort at time of recruitment were $43(12 \cdot 8)$ years and $11.5(9.5)$ years, respectively, with a male to female ratio of $3: 65$. These patients were studied prospectively for a total of 464 patient months (median 6, range 0-18), during which 233 separate assessments (median 3, 
range 1-10) were carried out. None of the patients was considered to have a concomitant viral or bacterial infection or condition other than SLE at time of assessment. Subsequent follow up supported this clinical opinion. Informed consent was obtained from each patient, and the protocol was approved by the Nottingham University Hospital Ethics Committee.

SLE disease activity was scored by a single observer (KLL) using the computerised BILAG index. A score of A or B in any of the eight organ systems assessed was considered to represent active disease. A flare in disease activity was defined as a change in BILAG index grade from inactive to active; disease remission was defined as a change from active to inactive on two successive assessments. A numerical score for each assessment (BILAG score) was also calculated from the individual scores for each organ system: A scored 9, B 3, $\mathrm{C} 1$, and $\mathrm{D}$ and $\mathrm{E}$ each scored 0 .

\section{LABORATORY MEASUREMENTS}

In all patients, venous blood was obtained at time of recruitment into this study for measurement of haemoglobin concentration, white cell count with differential count, platelet count, ESR, serum concentrations of urea and electrolytes, liver function tests, and concentrations of antibodies to nuclear antigens, antibodies to extractable nuclear antigens, anti-dsDNA, and plasma C3, C4 and C3d. An early morning urine sample was obtained at the same time for measurement of neopterin. ESR, plasma C3, C4 and $\mathrm{C} 3 \mathrm{~d}$, and urine neopterin were available also for all subsequent assessments.

Urine neopterin was measured, without prior knowledge of the BILAG status of the patients, using reverse phase high performance liquid chromatography (HPLC) as described previously ${ }^{7}$ and expressed in $\mu \mathrm{mol} / \mathrm{mol}$ creatinine. In brief, urine samples, protected from light and previously stored frozen, were centrifuged to remove debris, diluted 1 in 10 with water containing dimethylpterine as an internal standard and injected directly onto a Techsphere 5 ODS column (HPLC Technology Ltd). A binary gradient elution was used with an initial mobile phase of $2 \%$ methanol in $15 \mathrm{mmol} / \mathrm{l}$ phosphate buffer, $\mathrm{pH}$ $6 \cdot 4$, increasing to $25 \%$ methanol after 12 minutes, and neopterin was detected by its natural fluorescence $\left(\lambda_{\text {ex }} 353 \mathrm{~nm} ; \lambda_{\mathrm{em}} 438 \mathrm{~nm}\right)$. Urine creatinine was determined separately using a kinetic alkaline picrate (Jaffe) method; other test parameters of interest were quantified by standard methods: ESR by Seditainer ESR System, plasma C3 and C4 by nephelometry, and C3d by double decker immunodiffusion.

STATISTICAL ANALYSIS

Spearman's rank correlation coefficients $\left(r_{s}\right)$ were computed for various study parameters and the BILAG score. Differences between parameters within a group were evaluated with the Wilcoxon Matched Pairs Signed Rank Sum test. In order to avoid statistical bias, for patients who had more than one assessment during the study period we included only data from the initial assessment in the correlation analysis.

Urine neopterin measurements were made at varying intervals and over a period of months. In order to study the temporal relationship between urine neopterin concentration and SLE disease activity, the data set was therefore modelled as a time series. Initially, an analysis of covariance was carried out to assess the relationship between BILAG score and patient factors. In this model, time and patient were included as factors, urine neopterin as a covariate and BILAG score as the dependent variable. The initial assessment for each patient was numerically coded 0 , with subsequent consecutive assessments coded 1 , 2,3 , etc, and only patients with more than five assessments each were included into the model. Where time was found not to have a significant effect, the association between the mean BILAG score and the mean urine neopterin value for each patient was then examined by regression analysis. Similar analyses were carried out for ESR and plasma $\mathrm{C} 3, \mathrm{C} 4$ and $\mathrm{C} 3 \mathrm{~d}$. For these analyses, the SPSS $^{\circledR}$ software package was used; ${ }^{9} p<0.05$ was considered significant.

\section{Results}

Of the 68 patients, 35 had more than two assessments (nine had three, seven had four, five had five, four had six, seven had seven, one had eight and two had 10 assessments) during the study period. According to the BILAG index, 116 of these assessments were defined as active and 117 were inactive. Twenty five patients had active disease involving more than one organ system and 24 had active disease involving one organ system only. Active disease involved the general system in 10 patients, the mucocutaneous system in 24, the central nervous system in nine, the musculoskeletal system in 18 patients, the cardiorespiratory system in six, vasculitis in 12 patients, the renal system in three, and the haematological system in 17 patients, either in isolation or as part of multiple organ system disease.

At the time of initial evaluation, urine neopterin concentration was found to correlate significantly with the BILAG score $\left(r_{s}=0.32\right.$, $p=0.008)$. Significant correlations were also found for $\mathrm{C} 3\left(\mathrm{r}_{\mathrm{s}}=-0.38, \mathrm{p}=0.001\right)$, and C4 $\left(r_{s}=-0.45, p=0.0001\right)$, but not for ESR $\left(r_{s}=0.09\right)$ or C3d $\left(r_{s}=0.08\right)$.

URINE NEOPTERIN AND DISEASE FLARE

Fourteen patients had flares of their disease during the study period. Figure 1 shows plots of their respective urine neopterin values. Median urine neopterin value was found to be increased during the flare $(342 \mu \mathrm{mol} / \mathrm{mol}$ creatinine) compared with the inactive disease phase $(243 \mu \mathrm{mol} / \mathrm{mol}$ creatinine) (difference significant, $p=0.02$ ). Second successive 


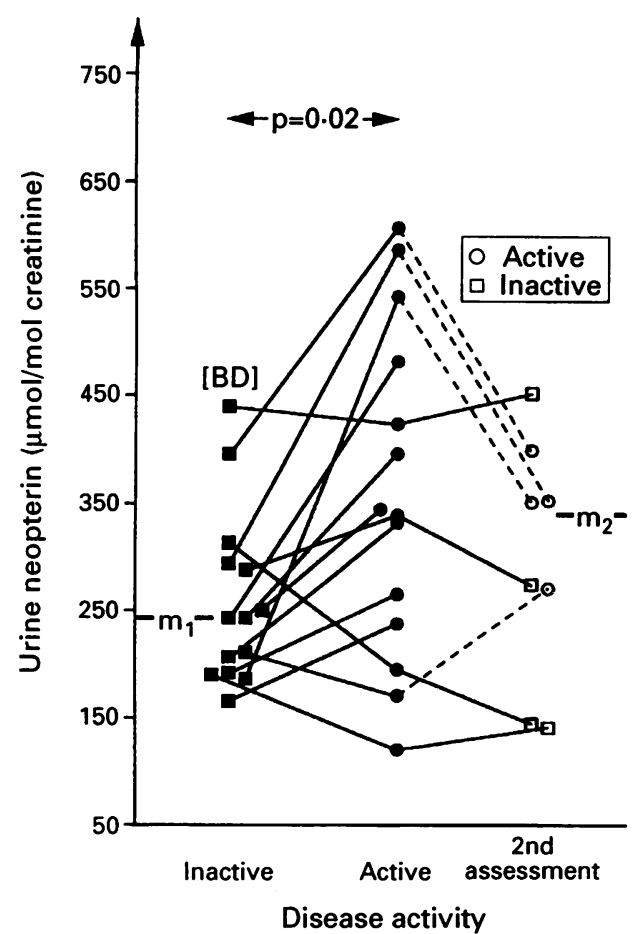

Figure 1 Comparison of direction of change in urine neopterin concentrations between successive assessments with disease activity in 14 patients who suffered flares of their disease. Second successive assessments were carried out, on average, 1.9 months (range 1-4 months) after the first. $m_{1}$ and $m_{2}=$ Median neopterin values for inactive and active disease phases, respectively. $B D=$ Patient referred to in text.

assessments were available for eight of the 14 patients; four were found to have persistently active disease with greater urine concentrations of neopterin compared with corresponding values during the inactive disease phase. Despite the disease remaining active, urine neopterin values in three of these four patients were lower in the second successive assessment compared with corresponding values during their disease flares, and these values corresponded with lower BILAG scores, suggesting clinical improvement. One patient (BD in fig 1) had persistent tiredness and lethargy with consistently increased urine neopterin concentrations in addition to increased C3d and ESR values, but the BILAG grade became active only when she developed acute pleurisy and a malar rash, which responded to subsequent treatment. Urine neopterin values for two patients showed a downward trend despite a flare in their disease that subsequently settled.

In contrast, no significant differences in ESR and plasma C3, C4 and C3d were found in these patients during their disease flares.

URINE NEOPTERIN AND DISEASE REMISSION Thirty patients with active disease went into remission during the study period (fig 2). Second successive assessments were available for 19 patients, 16 of whom remained in disease remission. Urine neopterin concentrations were significantly lower $(p=0.02)$ when the patients had inactive as opposed to active disease with median values of 249 and $320 \mu \mathrm{mol} / \mathrm{mol}$ creatinine, respectively. Urine neopterin values for the 16 patients who had

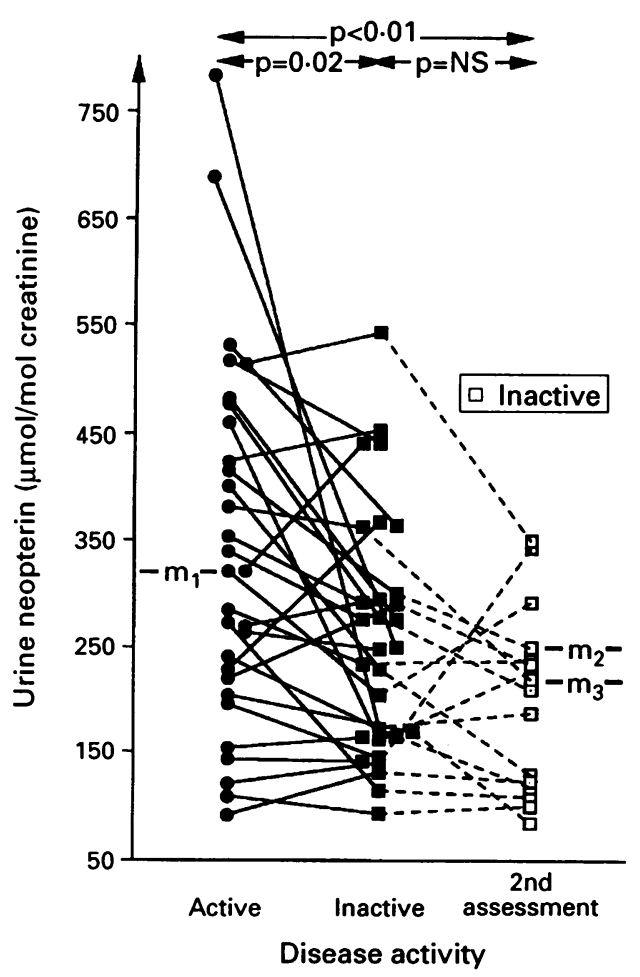

Figure 2 Comparison of direction of change in urine neopterin concentrations between successive assessments with disease activity in 30 patients with active disease who went into disease remission. Second successive assessments were carried out, on average, 2.9 months (range 1-6 months) after the first. $m_{1}, m_{2}$, and $m_{3}=$ Median neopterin values for active, inactive, and second successive inactive disease phases, respectively. NS = not significant. $a$

a second inactive assessment were also significantly lower than the corresponding values during the active disease phase $(p<0.01)$. In contrast, no significant differences were found between urine neopterin values for the two inactive assessments.

Significant differences $(p<0.01)$ were also found in ESR, plasma C4 and C3d values, but not plasma C3 values, in the active compared with the inactive phases for each of the patients.

\section{TIME SERIES ANALYSIS}

Figure 3 shows the serial plots of urine neopterin and BILAG score with time in 14 patients who completed more than five complete assessments each. In the analysis of covariance, with time and patient as factors, urine neopterin as a covariate and BILAG score as the dependent variable, time did not have a significant effect and could therefore be ignored in the multivariate model (table). Subsequent multiple regression analysis, ignoring the effect of time, showed that serial measurements of urine neopterin provided a significant predictor of the BILAG score

Analysis of covariance with urine neopterin as a covariate

\begin{tabular}{lccl}
\hline & Degrees of freedom & $F$ value & Probability $>F$ \\
\hline Urine neopterin & 1 & $10 \cdot 8$ & 0.002 \\
Time & 17 & $1 \cdot 4$ & $0 \cdot 186$ \\
Patient & 13 & $2 \cdot 3$ & 0.015 \\
Error & 67 & & \\
\hline
\end{tabular}



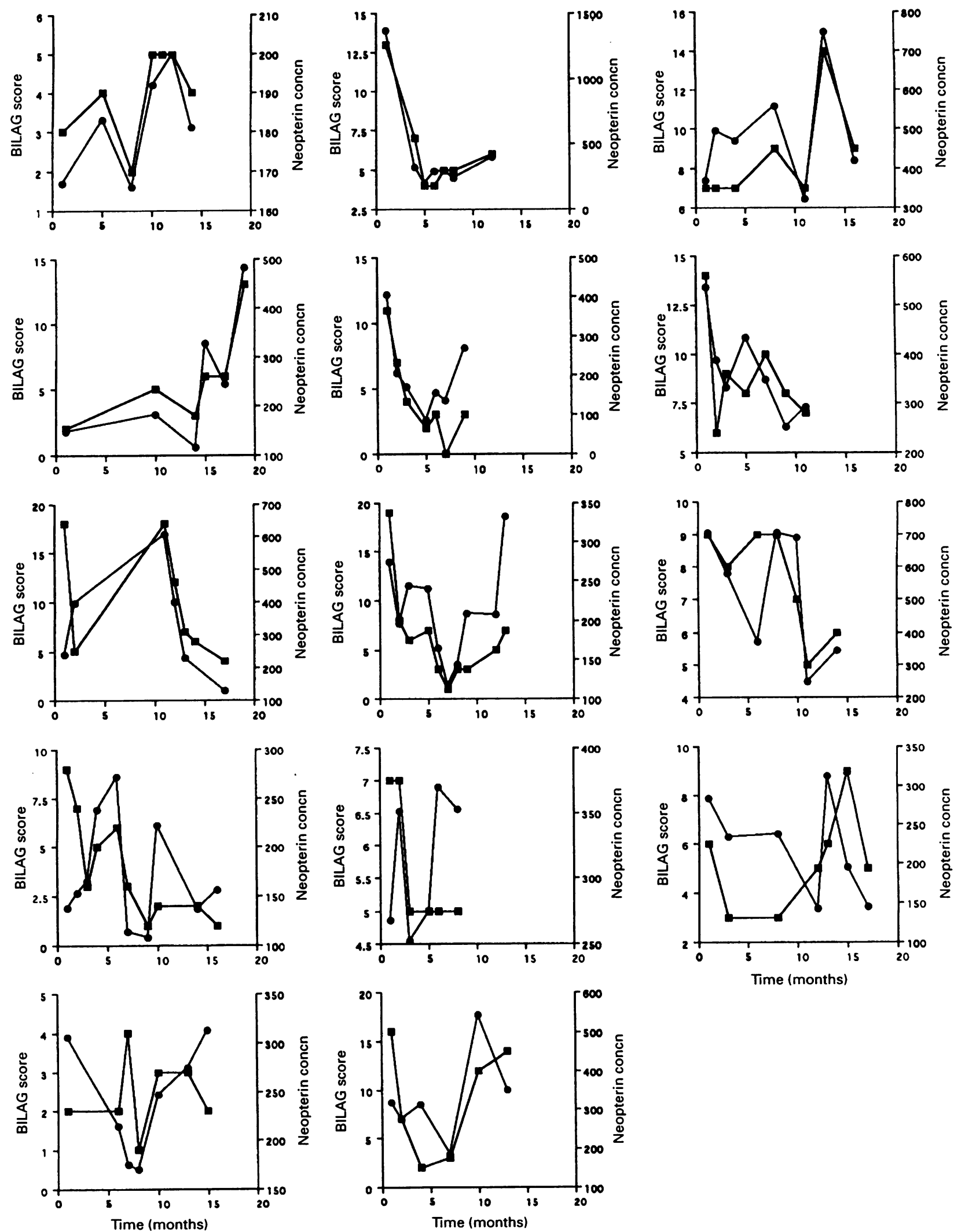

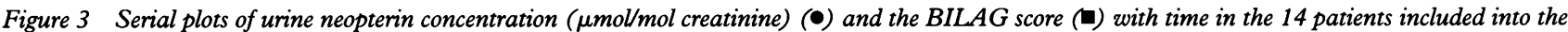
time series model.

(allowing for patient factors) $(r=0.6$, $\mathrm{p}<0.05)$.

In contrast, analyses of covariance of the relationship between the other test parameters (plasma C3, C4 and C3d, and ESR) and BILAG score, all revealed a significant effect of time $(p<0.05)$, but it was not feasible to define further this effect of time because of the relative paucity of the data sets.

\section{Discussion}

This study provides evidence that changes in urine concentrations of neopterin are significantly correlated with fluctuations in disease activity, scored using the BILAG index, among individual patients with SLE.

Single time point analysis showed that urine neopterin was significantly increased during flares in SLE disease activity. Similarly, a 
significant decrease in urine neopterin concentrations was observed in patients with active SLE who went into disease remission. In those patients whose disease remained active or in remission on second successive assessments, urine neopterin concentrations were found to be correspondingly greater or smaller compared with initial values. In addition, a significant correlation between urine neopterin concentration and BILAG score underlined the association of urine neopterin with clinical activity in SLE.

In the clinical setting, however, serial measurements of laboratory parameters are normally made to monitor patients. A number of studies have tried to address the temporal relationship between SLE disease activity and serum concentrations of complement, complement split products, and anti-dsDNA. ${ }^{1}{ }^{2} 10$ In those studies, only graphical presentations and single time point analyses were used to describe the data sets, though the study parameters were measured sequentially over a period of many months or years, leading to loss of sensitivity and the problem of dependence between test statistics. When clinical monitoring is based upon serial quantitative measurements, the detection and interpretation of abrupt changes in pattern of the data series are of paramount importance. Often, however, such series of data are difficult to interpret, as biological variations and errors arising in the collection, measurement and processing of the data and variations with time itself need to be taken into account. In this prospective study, we adopted time series modelling to investigate if serial measurements of urine neopterin could predict the BILAG score and hence SLE disease activity in individual patients.

The graphical plots of serial urine neopterin concentrations and BILAG score in individual patients, shown in figure 3 , suggest close relationships between the two measurements and with time. However, analysis of covariance indicated that the time variable added nothing to the prediction based on the urine neopterin covariate. Consequently, the time variable was ignored in the subsequent regression model. This showed a statistically significant association between serial concentrations of urine neopterin and BILAG score, with an $r$ value of $0.6(p<0.05)$. However, it should be noted that the entry point of each patient into the analysis was arbitrarily called "time zero", even though patient recruitment into the study took place over a period of months. This designation may have influenced the outcome of the time series modelling, but is inevitable in the clinical setting; the difficulty in analysing such data sets has been highlighted in other studies. ${ }^{11}$ Replication of these findings with a larger series of patients, using more regular measurements and a longer time frame, is required to validate our conclusions further.

None of the other study paramaters was found to mirror SLE disease activity as effectively as urine neopterin, using both single time point and time series analyses. Plasma $\mathrm{C} 3$ and $\mathrm{C} 4$ did not decrease significantly during SLE disease flares in individual patients, even though these two parameters correlated significantly with the BILAG score. In contrast, both ESR and plasma C3d failed to correlate significantly with BILAG score and their values in individual patients did not increase significantly during SLE disease flares. In corresponding analyses of covariance with serial measurements of ESR, plasma C3, C4, and $\mathrm{C} 3 \mathrm{~d}$ as covariates, time was found to have a significant effect on the BILAG score.

SLE is a chronic disease with variable activity characterised by numerous and varied abnormalities of the immune system, in particular upregulation of $\mathrm{T}$ lymphocytes. ${ }^{12}$ Recent attention has been focused on serum sIL-2 receptors, derived from surface bound IL-2 receptors on activated T lymphocytes in vivo, as a sensitive and more reliable index of clinical activity in patients with SLE and other rheumatic diseases. ${ }^{13}$ However, in our experience, there was considerable overlap between serum sIL-2 receptor values in SLE patients with active and inactive disease, ${ }^{4}$ suggesting that other factors, particularly renal function, must have an important influence on serum concentrations of sIL-2 receptors. ${ }^{14} 15$ Similarly, anti-dsDNA was found to be a poor discriminant of disease activity in our patient cohort. Thus neither sIL-2 receptor nor antidsDNA was included as parameters in this study. Neopterin is specifically produced by human macrophages when stimulated by interferon gamma released from activated $T$ lymphocytes, ${ }^{16}{ }^{17}$ and is therefore an indirect marker of the upregulation of the cellular immune system. Urine neopterin concentration has been shown to correlate significantly with serum sIL-2 receptor concentrations. ${ }^{18}$ It is, then, not surprising to find that urine neopterin is a more sensitive index of disease activity in SLE patients compared with established tests.

Our calculations were based on the BILAG index as the established index of SLE disease activity. The BILAG index is one of three currently available activity scales that have been shown to be reliable both between and within raters, ${ }^{19}$ the other two being Systemic Lupus Activity Measure (SLAM) ${ }^{20}$ and SLE Disease Activity Index (SLEDAI). ${ }^{21}$ High correlations between these three clinical activity scales have been demonstrated, suggesting that they are all measuring the same thing. ${ }^{22}$ Ideally, the reliability of our calculations should have been validated against another independent clinical activity scale. However, the inclusion of one or more of the study parameters in the other clinical activity scales makes them unsuitable for use as instruments to validate these as parameters of disease activity.

In conclusion, this prospective longitudinal study has indicated that serial urine concentrations of neopterin are significantly associated with SLE disease activity scored using the BILAG index. Measurement of urine neopterin is non-invasive, relatively simple and inexpensive and appears to be clinically useful for isolated assessments of disease activity in addition to serial monitoring of disease activity in the management of patients with SLE. 
The authors wish to thank Jim Hughes and Sandy Brown, QMC for performing the urine neopterin assays and Andrea Thomas, West Midlands Health Authority for computing assistance in the time series analyses.

1 ter Borg E T, Horst G, Hummer E J, Limburg P C Kallenberg C G M. Measurement of increases in antidouble-stranded DNA antibody levels as a predictor of disease exacerbations in systemic lupus erythematosus: long-term, prospective study. Arthritis Rheum 1990; 33: 634-43.

2 Swaak A J G, Aarden L A, Statius van Eps L, Feltkamp T E W. Anti-dsDNA and complement profiles as prognostic guides in systemic lupus erythematosus. Arthritis Rheum 1979; 22: 226-35

3 Wachter H, Fuchs D, Hausen A, Reibnegger G, Werner E R. Neopterin as marker for activation of cellular immunity: immunologic basis and clinical application. immunity: immunologic basis and

4 Lim K L, Jones A C, Brown N S, Powell R J. Urine neopterin as a parameter of disease activity in patients
with systemic lupus erythematosus: comparisons with with systemic lupus erythematosus: comparisons with
serum sIL-2R and antibodies to dsDNA, erythrocyte serum sIL-2R and antibodies to dsDNA, erythrocyte
sedimentation rate, and plasma $\mathrm{C} 3, \mathrm{C} 4$, and $\mathrm{C} 3$ sedimentation rate, and plasma C3, C4, and C3
degradation products. Ann Rheum Dis 1993; 52: 429-35. degradation products. Ann Rheum Dis 1993; 52: 429-35.
Symmons D P M, Coppock J S, Bacon P A, et al.

5 Symmons D P M, Coppock J S, Bacon P A, et al. clinical disease activity in systemic lupus erythematosus. $Q \mathcal{F}$ Med 1988; 258: 927-37.

6 Tan E M, Cohen A S, Fries J F, et al. The 1982 revised criteria for the classification of systemic lupus erythematosus. Arthritis Rheum 1982; 25: 1271-7.

7 Slazyk W E, Splerto F W. Liquid-chromatographic measurement of biopterin and neopterin in serum and measurement of biopterin and neopt

8 Crowder $M$, Hand D. Analysis of repeated measures. In Cox $\mathrm{D}$, Hinkley $\mathrm{D}$, Rubin $\mathrm{D}$, Silverman $\mathrm{B}$, eds.
Monographs on statistics and applied probability. Vol 41 . Monographs on statistics and applied

9 Statistical package for the social sciences". Chicago: SPSS 1992

10 Buyon J, Tamerius J, Belmont $\mathrm{H} \mathrm{M}$, Abramson S Assessment of disease activity and impending flare in patients with systemic lupus erythematosus: comparison of the use of complement split products and conventional measurements of complement. Arthritis Rheum 1992; 35: 1028-37.

11 Smith A F M, West M. Monitoring renal transplants: an application of the multiprocess Kalman filter. Biometric application of the

12 Via C S, Handwerger B S. T-cell and B-cell function in lupus. Curr Opin Rheumatol 1992; 4: 630-4.

13 Rubin LA. The soluble interleukin-2 receptor in rheumatic disease. Arthritis Rheum 1990; 33: 1145-8.

14 Nelson D L, Wagner D K, Marcon L, et al. An analysis of soluble IL-2 receptors in human neoplastic disorders. In Albertini A, Lentant C, Poeletti R, eds. Biotechnology in clinical medicine. New York; Raven Press, 1987.

15 Manoussakis M N, Papadopoulos G K, Drosos A A Moutsopoulos H M. Soluble interleukin 2 receptor molecules in the serum of patients with autoimmune diseases. Clin Immunol Immunopathol 1989; 50: 321-32.

16 Huber C, Fuchs D, Hausen A, et al. Pteridines as a new marker to detect human T cells activated by allogeneic or determinants. F Immunol 1983; 130: 1047-50.

17 Huber C, Batchelor J R, Fuchs D, et al. Immune responseassociated production of neopterin. Release from macrophages primarily under control of interferongamma. $\mathcal{F}$ Exp Med 1984; 160: 310-6.

18 Lim K L, Atta M, Hughes J, Brown N S, Powell R J. Serum levels of soluble interleukin 2 receptor (IL-2R) and urine neopterin with disease activity in patients with systemic lupus erythematosus (SLE). Arthritis Rheum 1992; 35: S209.

19 Hay E, Gordan C, Emery P. Assessment of lupus; where are we now? Ann Rheum Dis 1993; 52: 169-72.

20 Liang M, Socher S, Roberts W, Esdaile J. Measurement of systemic lupus erythematosus activity in clinical research.

21 Bombardier C, Gladman D D, Urowitz M B, Caron D, Chang $\mathrm{C} \mathrm{H}$. Derivation of the SLEDAI. A disease activity index for lupus patients. The Committee on Prognosis Studies in SLE. Arthritis Rheum 1992; 35: 630-40.

22 Gladman D D, Goldsmith C H, Urowitz M B, et al. Crosscultural validation and reliability of 3 disease activity indices in systemic lupus erythematosus. 7 Rheumatol 1992; 19: 608-11. 